\title{
IMAGEM CORPORAL E AUTOESTIMA EM IDOSOS: UMA REVISÃO INTEGRATIVA DA LITERATURA
}

\author{
Sedinei Lopes Copatti ${ }^{1}$ \\ Adriana Gracietti Kuczmainski² \\ Fátima Ferretti ${ }^{3}$ \\ Clodoaldo Antônio De Sá4
}

resumo

Trata-se de uma revisão integrativa que teve como objetivo analisar as produções científicas publicadas sobre imagem corporal e autoestima em idosos. A pesquisa foi realizada por meio da busca direta de artigos completos na Biblioteca Virtual em Saúde, resultando num total de 24 artigos. Destes, constituiu-se uma amostra final composta de 14 artigos, os quais atenderam os critérios para inclusão no estudo.

1 Graduado em Educação Física, Mestre em Ciências da Saúde, Pesquisador vinculado ao grupo de pesquisa VITA da Universidade Comunitária da Região de Chapecó (Unochapecó). E-mail: sedinei@ unochapeco.edu.br.

2 Graduada em Enfermagem, Mestre em Ciências da Saúde, Professora da Universidade do Estado de Santa Catarina (UDESC) do curso de graduação em Enfermagem. E-mail: adriana.kuczmainski@ udesc.br.

3 Graduada em Fisioterapia, Doutora em Saúde Coletiva, Professora da Universidade Comunitária da Região de Chapecó (Unochapecó), vinculada ao programa de Pós-graduação em Ciências da Saúde.E-mail: ferrettifisio@yahoo.com.br.

4 Graduado em Educação Física, Doutor em Ciência do Movimento Humano, Professor da Universidade Comunitária da Região de Chapecó (Unochapecó), vinculado ao programa de Pós-graduação em Ciências da Saúde. E-mail: clododoaldodesa@gmail.com. 
A análise possibilitou a subdivisão dos trabalhos selecionados em dois eixos temáticos: a) exercício físico e sua relação com a imagem corporal e autoestima; b) fatores associados à imagem corporal e autoestima em idosos. Por fim, este estudo evidenciou a prática de exercício físico como uma atividade de influência positiva na percepção da imagem corporal e da autoestima dos idosos. Entre os fatores associados à imagem corporal e autoestima, destaca-se a maior insatisfação do sexo feminino e o comprometimento da autopercepção corporal e autoestima em idosos em condições clínicas de adoecimento e tratamento de saúde. Ao final, a presente revisão contribui para a produção científica sobre a temática, servindo como base instrumentalizar os profissionais para uma abordagem multidimensional e de qualidade na atenção à população idosa.

palavras-chave

Envelhecimento. Atividade Física. Autopercepção. Saúde.

\section{Introdução}

Imagem corporal refere-se à experiência corporal que as pessoas têm sobre sua própria aparência e funcionamento de seu corpo (ALMEIDA; LOUREIRO; SANTOS, 2002) e ao reconhecimento que o indivíduo faz de si mesmo, considerando seus sentimentos, potencialidades, atitudes e ideias (PINQUART; SÖRENSEN, 2001). A capacidade avaliativa do sujeito a respeito de seu próprio corpo relaciona-se diretamente com a autoestima, compreendida como o sentimento, a consideração e o apreço que a pessoa tem por si própria (ROSENBERG, 1965). Para Magalhães et al. (2008), a imagem corporal apresenta-se como um dos componentes envolvidos no conceito de autoestima, sendo interligadas e dependentes uma da outra.

Segundo Erthal (1986), a imagem de si está sempre em mudança, conforme as experiências vivenciadas no decorrer do tempo. Mais especificamente em relação aos idosos, as experiências adquiridas ao longo da vida ligadas a imagem corporal, são marcadas pela cultura, sociedade e grupos nos quais o indivíduo encontra-se inserido e o papel social que o sujeito exerce nesses contextos (SILVA; CAMINHA; GOMES, 2013). Nesse sentido, Pinquart e Sörensen (2001) apontam que a interação entre esses ambientes de vivência se apresenta como importante base para a composição da imagem corporal e 
percepção do corpo pelo próprio indivíduo. Na sociedade atual, caracterizada como um cenário de construção e imposição de modelos estéticos, na qual o ideal corporal predominante é caracterizado pela jovialidade (SAIKALI et al., 2004), o processo de envelhecimento muitas vezes é visto a partir de uma visão preconceituosa, como pernicioso e, frequentemente, associado a significados e imagens negativas, fato que interfere negativamente na forma como o sujeito se percebe ao longo do processo de envelhecimento (GUERRA; CALDAS, 2010).

Apesar da pertinência e atualidade da temática, além de considerar os critérios de busca e seleção, esta revisão encontrou apenas 14 artigos que abordaram a questão da imagem corporal e autoestima em idosos. Esses artigos refletem, sobretudo, a negação da velhice, condição associada a imagem que os idosos fazem de si mesmos, tornando-os mais propensos a sentimentos de autodesvalorização e de baixa autoestima (NETTO, 2002; ROCHA; CUNHA, 1994). Por outro lado, esta revisão também evidenciou que o idoso, quando comparado a outras faixas etárias, apresenta-se mais satisfeito quanto a sua imagem corporal (DANTAS, 1994; CHAIM; IZZO; SERA, 2009).

Diante desse contexto, conhecer a relação do idoso com seu corpo e como isso afeta sua autopercepção e autoestima, apresenta-se como uma questão fundamental na compreensão do processo de envelhecimento. Da mesma forma, conhecer e identificar experiências e modelos de intervenções que se mostraram eficientes na melhoria da experiência corporal de envelhecer afetando positivamente o sentimento, consideração e o apreço do idoso por si mesmo, constitui um conhecimento fundamental para os profissionais de saúde e cuidadores, com vistas a qualificar o processo de cuidado e a relação com o idoso, contribuindo de fato para um envelhecimento bem-sucedido. Nesse sentido, esse estudo teve como objetivo analisar as produções científicas publicadas sobre imagem corporal e autoestima em idosos.

\section{Método}

Trata-se de uma revisão integrativa de literatura, a qual caracteriza-se como uma metodologia que proporciona a síntese do conhecimento sobre o estado da arte quanto a um determinado tópico (GANONG, 1987). Essa pesquisa foi conduzida a partir das seguintes etapas descritas por Ganong (1987): a) identificação do tema e elaboração da questão de pesquisa; b) estabelecimento de critérios de exclusão e inclusão; c) seleção da amostra; d) definição das informações a serem extraídas dos estudos selecionados/categorização e organização dos dados em tabela; e) avaliação dos estudos incluídos na revisão; 
f) análise e discussão dos resultados e apresentação do estudo em forma de artigo científico. A revisão orientou-se a partir de um protocolo de pesquisa, devidamente validado por uma pesquisadora com experiência na temática.

Foram incluídos na presente revisão trabalhos publicados no formato de artigos científicos (artigos originais, revisões sistematizadas, relatos de experiências, ensaios teóricos, reflexões), nos idiomas: português, inglês e espanhol e que incluíram, no título, os descritores do estudo. Foram excluídos os estudos duplicados e àqueles envolvendo sujeitos submetidos a tratamentos estéticos ou que incluíam amputações ou tratamentos envolvendo implantes de cunho estético ou funcional.

Para o levantamento dos artigos, utilizou-se a base de dados da Biblioteca Virtual em Saúde e os descritores foram selecionados a partir da terminologia em saúde consultada nos Descritores em Ciências da Saúde (DeCS). A busca na referida base de dados foi realizada a partir do cruzamento dos seguintes descritores: "imagem corporal" AND "idosos"; "autoestima" AND "idosos", "idoso" AND "imagem corporal", "idoso" AND "autoestima", "idoso" AND "autoimagem", "autoimagem" AND "idosos", selecionando o "método integrado", "todos os índices", "todas as fontes" e "palavras no título".

A seleção dos trabalhos foi realizada em setembro de 2014. A busca gerou 24 artigos. Após aplicação dos critérios de exclusão, sete artigos foram excluídos por duplicidade e três trabalhos foram excluídos por estarem fora da temática estudada. Em agosto de 2015 a busca foi refeita e gerou apenas um artigo que foi publicado após a data da primeira busca, contudo o mesmo não tem relação direta com a pergunta do estudo e dessa forma não foi incluído no trabalho. Assim, constituiu-se a amostra final desta revisão em 14 artigos científicos, cujo protocolo de busca e seleção está representado na figura 1. 
Figura 1 - Fluxograma de seleção dos estudos que compuseram a revisão integrativa.

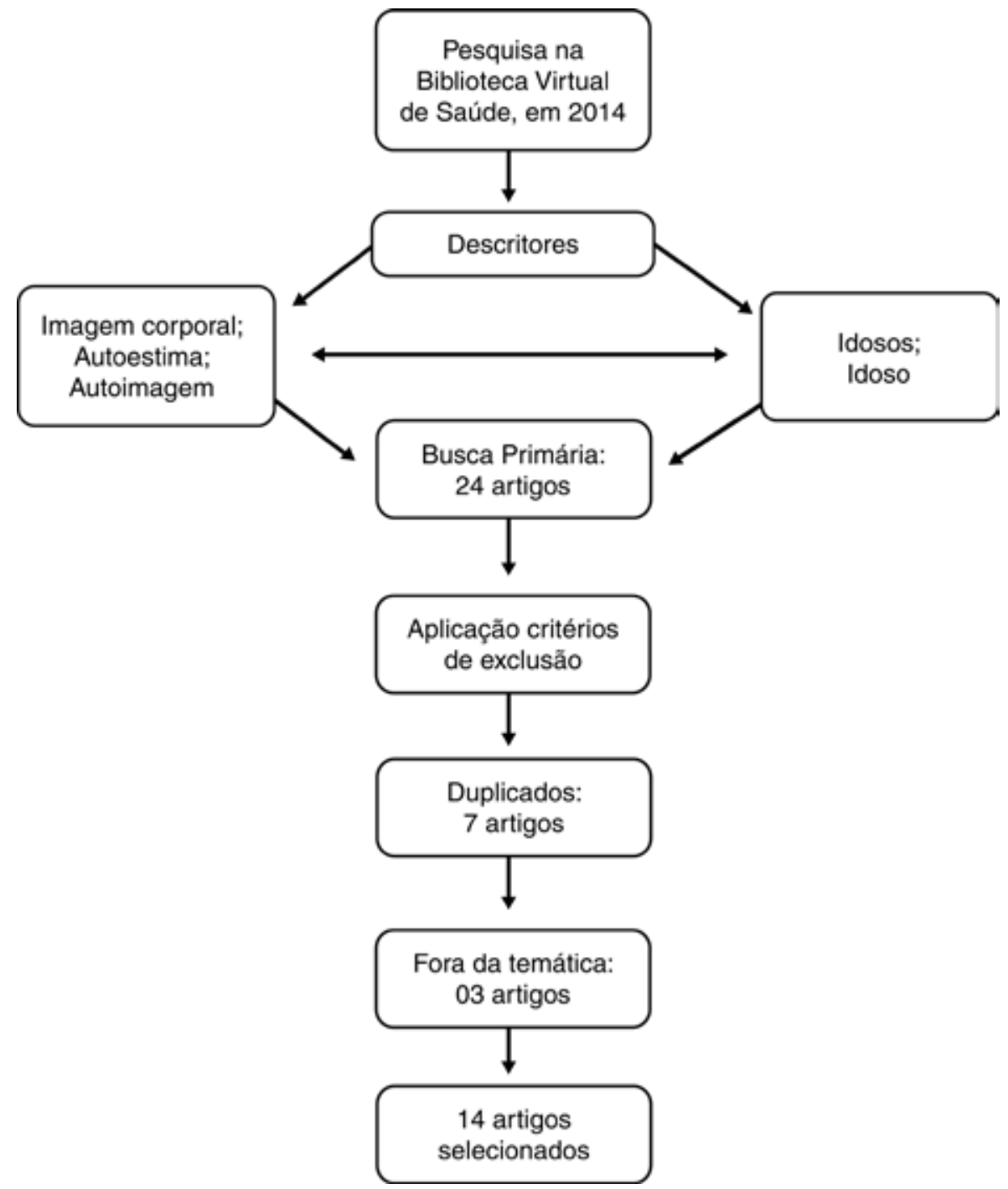

Fonte: Figura elaborada pelos autores. 
Após a leitura dos 14 artigos, identificou-se 13 artigos originais e uma revisão de literatura. Entre os artigos, 12 eram de corte transversal e um com prática de intervenção, sendo apenas um destes publicado em língua estrangeira (inglês). A síntese dos estudos analisados pode ser visualizada na tabela 1.

Tabela 1 - Síntese dos estudos analisados nesta revisão envolvendo imagem corporal e autoestima de idosos.

\begin{tabular}{|c|c|c|}
\hline Autor/Ano & Características do estudo & Principais resultados \\
\hline $\begin{array}{l}\text { Meurer et al. } \\
\text { (2012) }\end{array}$ & $\begin{array}{l}\text { Tipo de estudo: Transversal. } \\
\text { Amostra: } 67 \text { mulheres e } 16 \text { homens, com } \\
\text { idade entre } 60 \text { e } 88 \text { anos. } \\
\text { Variáveis analisadas: Características } \\
\text { demográficas, socioeconômicas, sinto- } \\
\text { mas depressivos e fatores motivacionais, } \\
\text { autoestima. } \\
\text { Coleta de dados: Escala de Rosenberg, } \\
\text { inventário de motivação para a prática regu- } \\
\text { lar de atividades físicas. }\end{array}$ & $\begin{array}{l}\text { Relação inversa entre autoestima } \\
\text { e sintomas de depressão; } \\
\text { Baixa ocorrência de sintomas } \\
\text { depressivos; } \\
\text { Elevada autoestima; } \\
\text { Elevada motivação; } \\
\text { Influência positiva do exercício } \\
\text { na pontuação das variáveis } \\
\text { estudadas. }\end{array}$ \\
\hline $\begin{array}{l}\text { Benedetti, } \\
\text { Petroski, Gon- } \\
\text { çalves (2003) }\end{array}$ & $\begin{array}{l}\text { Tipo de estudo: Intervenção (programa de } \\
\text { exercício físico). } \\
\text { Amostra: } 29 \text { mulheres com idade = ou > } \\
60 \text { anos. } \\
\text { Variáveis analisadas: Autoimagem e } \\
\text { autoestima } \\
\text { Coleta de dados: Questionário proposto } \\
\text { por Steglich (1978). }\end{array}$ & $\begin{array}{l}\text { Melhora autoestima grupo } \\
\text { experimental; } \\
\text { Melhora autoimagem grupo } \\
\text { controle. }\end{array}$ \\
\hline $\begin{array}{l}\text { Mazo, Car- } \\
\text { doso, Aguiar } \\
\text { (2006) }\end{array}$ & $\begin{array}{l}\text { Tipo de estudo: Transversal } \\
\text { Amostra: } 132 \text { mulheres e } 33 \text { homens com } \\
\text { idade >60 anos. } \\
\text { Variáveis analisadas: Autoestima, } \\
\text { percepção de saúde e aspectos } \\
\text { sociodemográficos. } \\
\text { Coleta de dados: Escala de Rosenberg } \\
\text { (1965). }\end{array}$ & $\begin{array}{l}\text { Elevada autoestima: em 65\% } \\
\text { dos idosos; } \\
\text { Elevada autoimagem: em 82\% } \\
\text { dos idosos. }\end{array}$ \\
\hline $\begin{array}{l}\text { Antunes, Mazo, } \\
\text { Balbé (2011) }\end{array}$ & $\begin{array}{l}\text { Tipo de estudo: Transversal } \\
\text { Amostra: } 53 \text { mulheres e } 7 \text { homens com } \\
\text { idade média de } 69,5 \text { anos }(\mathrm{SD}=5,9) \text {. } \\
\text { Variáveis analisadas: Autoestima, } \\
\text { autoimagem. } \\
\text { Coleta de dados: Questionário proposto } \\
\text { por Steglich (1978). }\end{array}$ & $\begin{array}{l}\text { Percepção positiva da idade: } \\
62,4 \% \text { dos idosos; } \\
\text { Elevada autoestima indepen- } \\
\text { dente do sexo e da faixa etária; } \\
\text { Associação positiva entre avalia- } \\
\text { ção de saúde e autoestima } \\
\text { para ambos os sexos; } \\
\text { Autoestima não foi associada } \\
\text { com escolaridade, estado civil } \\
\text { e renda mensal familiar; } \\
\text { Maior autoestima entre homens } \\
\text { com } 70 \text { anos ou mais em rela- } \\
\text { ção a homens mais jovens. }\end{array}$ \\
\hline
\end{tabular}




\begin{tabular}{|c|c|c|}
\hline $\begin{array}{c}\text { Meurer, Bene- } \\
\text { detti, Mazo } \\
\text { (2011) }\end{array}$ & $\begin{array}{l}\text { Tipo de estudo: Transversal } \\
\text { Amostra: } 90 \text { mulheres e } 21 \text { homens } \\
\text { com idade entre } 60 \text { e } 88 \text { anos. } \\
\text { Variáveis analisadas: Fatores motivacio- } \\
\text { nais e autoestima. } \\
\text { Coleta de dados: Escala de Rosenberg } \\
\text { (1965), Inventário de motivação para a prá- } \\
\text { tica regular de atividades físicas, American } \\
\text { Alliance for Health, Physical Education, } \\
\text { Recreation and Dance (AAHPERD). }\end{array}$ & $\begin{array}{l}\text { Menor autoestima em idosos } \\
\text { com doenças em relação } \\
\text { aos sem doença; } \\
\text { ldosos com mais de um ano } \\
\text { de participação no programa } \\
\text { apresentaram maior autoestima } \\
\text { que os que participam a menos } \\
\text { de um ano; } \\
\text { Não houve associação entre } \\
\text { autoestima e fatores motiva- } \\
\text { cionais (controle de estresse, } \\
\text { saúde, sociabilidade, competitivi- } \\
\text { dade, estética e prazer). }\end{array}$ \\
\hline $\begin{array}{l}\text { Chaim, Izzo, } \\
\text { Sera (2009) }\end{array}$ & $\begin{array}{l}\text { Tipo de estudo: Transversal } \\
\text { Amostra: } 26 \text { mulheres ( } 82,9 \pm 3,5 \text { anos) e } \\
15 \text { homens ( } 70,5 \pm 5,8 \text { anos). } \\
\text { Variáveis analisadas: Imagem corporal e } \\
\text { autoestima. } \\
\text { Coleta de dados: Imagem corporal pela } \\
\text { Escala de Sorensen e Stunkard; Escala } \\
\text { de Rosenberg (1965) para avaliação da } \\
\text { autoestima. }\end{array}$ & $\begin{array}{l}\text { Maioria dos idosos insatisfeitos } \\
\text { com imagem corporal; } \\
\text { ldosos apresentaram elevada } \\
\text { autoestima. }\end{array}$ \\
\hline $\begin{array}{l}\text { Santos et al. } \\
\text { (2012) }\end{array}$ & $\begin{array}{l}\text { Tipo de estudo: Transversal } \\
\text { Amostra: } 156 \text { mulheres e } 48 \text { homens com } \\
\text { idade entre } 60 \text { e } 82 \text { anos. } \\
\text { Variáveis analisadas: Percepção de } \\
\text { estresse, sintomas depressivos e autoes- } \\
\text { tima em idosos. } \\
\text { Coleta de dados: Escala de Estresse } \\
\text { Percebido (EEP), Escala de Depressão } \\
\text { Geriátrica (GDS), Escala de Autoestima de } \\
\text { Rosenberg (EAE) e Memory Assessment } \\
\text { Complain Questionnaire (MAC-Q). }\end{array}$ & $\begin{array}{l}\text { Idosos com queixas de com- } \\
\text { prometimento de memória } \\
\text { apresentaram maiores níveis de } \\
\text { estresse percebido e depressão } \\
\text { e menores de autoestima; } \\
\text { Correlação negativa entre } \\
\text { escore de queixas de memória e } \\
\text { estresse percebido. }\end{array}$ \\
\hline $\begin{array}{c}\text { Brandão et al. } \\
\text { (2004) }\end{array}$ & $\begin{array}{l}\text { Tipo de estudo: Transversal } \\
\text { Amostra: } 7 \text { mulheres e } 5 \text { homens com } \\
\text { idade superior a } 60 \text { anos. } \\
\text { Variáveis analisadas: Avaliação autorrefe- } \\
\text { rida da imagem corporal. } \\
\text { Coleta de dados: A Escala de Imagem } \\
\text { Corporal/BIS - Body Image Scale. }\end{array}$ & $\begin{array}{l}\text { Dificuldade significativa ao } \\
\text { abordar aspectos inconscientes } \\
\text { da representação da imagem } \\
\text { corporal frente à doença; } \\
\text { Idosos apoiaram-se no discurso } \\
\text { religioso e na crença de que a } \\
\text { doença teria cura, bem como no } \\
\text { desejo de desconhecimento da } \\
\text { doença. }\end{array}$ \\
\hline $\begin{array}{c}\text { Menezes et al. } \\
\text { (2014) }\end{array}$ & $\begin{array}{l}\text { Tipo de estudo: Transversal } \\
\text { Amostra: } 542 \text { mulheres e } 241 \text { homens } \\
\text { com idade entre } 60 \text { a } 103 \text { anos. } \\
\text { Variáveis analisadas: Imagem corporal, } \\
\text { grupo etário, estado nutricional, número de } \\
\text { doenças, percepção da saúde, prática de } \\
\text { atividade física. } \\
\text { Coleta de dados: Escala de nove silhuetas } \\
\text { de Stunkard. }\end{array}$ & $\begin{array}{l}\text { O sexo masculino apresentou } \\
\text { maior satisfação com a imagem } \\
\text { corporal quando comparados } \\
\text { com o sexo feminino; Idosos } \\
\text { mais velhos do sexo masculino } \\
\text { apresentaram-se mais satisfeitos } \\
\text { que idosos mais jovens; O baixo } \\
\text { peso e sobrepeso estavam rela- } \\
\text { cionados a um risco aumentado } \\
\text { de insatisfação no sexo feminino. }\end{array}$ \\
\hline
\end{tabular}




\begin{tabular}{|c|c|c|}
\hline Autor/Ano & Características do estudo & Principais resultados \\
\hline $\begin{array}{c}\text { Rocha, } \\
\text { Terra } \\
\text { (2013) }\end{array}$ & $\begin{array}{l}\text { Tipo de estudo: Revisão de literatura. } \\
\text { Amostra: } 54 \text { artigos científicos } \\
\text { Variáveis analisadas: Percepção da } \\
\text { imagem corporal, envelhecimento, idosos e } \\
\text { ageismo. } \\
\text { Coleta de dados: Bases de dados } \\
\text { Medline/PubMed e Lilacs. }\end{array}$ & $\begin{array}{l}\text { Os autores encontraram asso- } \\
\text { ciações entre a imagem corporal } \\
\text { e saúde mental em idosos; A } \\
\text { autoestima está associada à } \\
\text { preocupação com a imagem } \\
\text { corporal; A insatisfação com a } \\
\text { imagem corporal tem sido rela- } \\
\text { cionada a distúrbios psicológicos } \\
\text { e depressão. }\end{array}$ \\
\hline $\begin{array}{l}\text { M. Guedes, } \\
\text { H. Guedes, } \\
\text { Almeida } \\
\text { (2011) }\end{array}$ & $\begin{array}{l}\text { Tipo de estudo: Transversal } \\
\text { Amostra: } 22 \text { mulheres com idade } \\
\text { acima de } 60 \text { anos } \\
\text { Variáveis analisadas: Autoimagem } \\
\text { Coleta de dados: Entrevistas semiestrutu- } \\
\text { radas associados a momentos } \\
\text { de observação. }\end{array}$ & $\begin{array}{l}\text { Trabalhos manuais fortalece- } \\
\text { ram a autoimagem das idosas; } \\
\text { Trabalhos manuais minimizam } \\
\text { aspectos decorrentes do enve- } \\
\text { Ihecimento; Trabalhos manuais } \\
\text { permite surgir habilidades artísti- } \\
\text { cas e o seu aprimoramento. } \\
\end{array}$ \\
\hline $\begin{array}{c}\text { Silva, Santos, } \\
\text { Berardinelli } \\
(2010)\end{array}$ & $\begin{array}{l}\text { Tipo de estudo: Transversal } \\
\text { Amostra: } 11 \text { idosos (Não especifica sexo } \\
\text { e idade). } \\
\text { Variáveis analisadas: Imagem corporal e } \\
\text { autocuidado. } \\
\text { Coleta de dados: Pesquisa Dinâmica do } \\
\text { Corpo como Território Mínimo (DCTM). }\end{array}$ & $\begin{array}{l}\text { Imagem corporal se associou } \\
\text { com mudanças impostas } \\
\text { pelo envelhecimento, que, por } \\
\text { sua vez, podem até afetar o } \\
\text { senso de identidade do idoso; } \\
\text { Os idosos manifestaram sua } \\
\text { preocupação com o descuidado } \\
\text { com o corpo, ressaltando a } \\
\text { vulnerabilidade para as } \\
\text { doenças crônicas. }\end{array}$ \\
\hline $\begin{array}{c}\text { Pracidelli et } \\
\text { (2001) }\end{array}$ & $\begin{array}{l}\text { Tipo de estudo: Transversal } \\
\text { Amostra: } 9 \text { mulheres e } 4 \text { homens com } \\
\text { idade entre } 62-93 \text { anos. } \\
\text { Variáveis analisadas: Imagem corporal. } \\
\text { Coleta de dados: Desenhos-Estória com } \\
\text { Tema e entrevistas. semidirigidas. }\end{array}$ & $\begin{array}{l}\text { A aplicação de tratamento } \\
\text { conjunto entre fisioterapia e psi- } \\
\text { cologia permitiu uma maior cons- } \\
\text { cientização de si mesmo por } \\
\text { parte do paciente, deixando de } \\
\text { idealizar imagens, prender-se ao } \\
\text { passado e, consequentemente, } \\
\text { abandonando estereótipos. }\end{array}$ \\
\hline
\end{tabular}

Em relação aos artigos originais, cinco foram realizados com idosos participantes de grupos de atividade física, quatro com idosos hospitalizados, dois apresentaram características populacionais, um em grupo de convivência e um em asilo. Foram encontrados seis artigos que analisaram a influência da prática atividades e/ou exercícios físicos na percepção da imagem corporal e autoestima em idosos. Cabe destacar que esses artigos foram publicados por pesquisadores de duas universidades públicas de Florianópolis/SC e, que a amostra de cinco dos seis estudos foi composta por dois grupos de idosos.

Meurer, Benedetti e Mazo (2011) relatam uma associação significativa entre autoestima e presença de doença, na qual idosos saudáveis participantes 
do programa Floripa Ativa - Fase B apresentaram melhor autoestima quando comparados com idosos doentes. Outro resultado importante desse estudo foi o fato de que o tempo de participação no programa voltado ao aumento de força, equilíbrio, flexibilidade, coordenação e resistência aeróbia se relacionou diretamente com o aumento da autoestima.

Meurer et al. (2012) estudaram a relação entre autoestima, sintomas depressivos e motivacionais de idosos participantes do programa Floripa Ativa - fase B e observaram que a autoestima elevada possui relação direta com o baixo número de sintomas relacionados à depressão. Outro achado importante se refere a fatores motivacionais, dos quais apenas a busca pela estética não se destacou entre os idosos investigados. Benedetti, Petroski e Gonçalves (2003) observaram que um programa de exercício físico sistemático realizado por idosos de uma instituição asilar, afetou positivamente a autoestima do grupo experimental e, de maneira indireta, a autoimagem do grupo controle.

Mazo, Cardoso e Aguiar (2006) estudaram as relações da autoestima e autoimagem com os fatores que levam a adesão e permanência no programa de hidroginástica do Grupo de Estudos da Terceira Idade (GETI) da Universidade do Estado de Santa Catarina (UDESC). Melhora na condição física, social e psicológica motivam tanto a adesão quanto a permanência do grupo de ginástica, além disso, evidenciou-se uma relação positiva entre fatores de permanência no grupo com o aumento da autoimagem.

Meurer, Benedetti e Mazo (2009) identificaram que a participação de idosos em grupos de atividade física promove a autoestima e a autoimagem. Fatores como a condição financeira, e satisfação com a vida que se tem também foram abordados. Observou-se que os níveis socioeconômicos elevados estão associados com elevada autoestima. Outra observação feita pelos autores foi à superioridade das atividades em grupo sobre as atividades individuais na melhora da autoestima e autoimagem dos idosos.

A fim de avaliar a percepção da imagem corporal de idosos internados no Central do Hospital das Clínicas da Faculdade de Medicina da Universidade de São Paulo (HCFMUSP), Pracidelli et al. (2001) observaram dificuldade de aceitação do processo de envelhecimento nos pacientes estudados. Após uma intervenção multidisciplinar envolvendo profissionais de fisioterapia e psicologia, na qual utilizou-se procedimentos fisioterápicos de acordo com as necessidades de cada paciente além da técnica de expressão gráfica auto perceptiva, observou-se, mudanças positivas na percepção da imagem corporal dos idosos.

Brandão et al. (2004) afirmam que existe uma dificuldade significativa de identificar aspectos inconscientes da representação da imagem corporal frente à doença, condição observada em estudo realizado no ambulatório 
de cuidados paliativos do HCFMUSP, o qual buscou identificar a avaliação autorreferida da imagem corporal de pacientes idosos com diagnóstico de câncer, sem possibilidade terapêutica de cura. Através do uso de escalas de auto avaliação da imagem corporal e da autoestima, Chaim, Izzo e Sera (2009) observaram significativa insatisfação do grupo estudado, visto que gostariam de silhuetas mais magras. Apesar da insatisfação com a imagem corporal os idosos apresentaram autoestima elevada.

Santos et al. (2012) apontam que idosos que relataram queixa subjetiva de comprometimento de memória apresentaram maior estresse, mais sintomas de depressão e baixa autoestima em comparação aos idosos sem queixa. Menezes et al. (2014) demonstram associação estatisticamente significativa entre percepção da imagem corporal e grupo etário e estado nutricional. Entre os homens, idades avançadas constituíram fator de proteção para insatisfação. E, no geral, homens apresentaram maior satisfação com a imagem corporal quando comparados às mulheres e a insatisfação das mulheres foi relacionada ao baixo peso e sobrepeso.

Ao realizar um estudo em um grupo de convivência M. Guedes, H. Guedes e Almeida (2011) evidenciaram que trabalhos manuais fortaleceram a autoimagem das idosas participantes do estudo, através da concretização dos trabalhos, mas significativamente considerando a socialização resultante das atividades em grupo, referida pelas participantes do estudo, como uma extensão da família. Um estudo foi realizado com um grupo de universitários da terceira idade, no qual Silva, Santos e Berardinelli (2010) apontam que o autocuidado com o corpo exige do indivíduo conhecimentos e também a observação de si próprio, para a tomada de consciência daquilo que lhe faz mal nos seus hábitos de vida, de agir e de pensar.

Em revisão de literatura, Rocha e Terra (2013) encontraram associações entre a imagem corporal e à saúde mental em idosos. A autoestima está associada à preocupação com a imagem corporal e a insatisfação com a imagem corporal tem sido relacionada a distúrbios psicológicos e depressão.

Observou-se uma preferência pelos instrumentos de avaliação de autoestima e imagem corporal, na qual foi encontrado cinco artigos utilizando a escala de avaliação de Rosenberg e três artigos utilizando o questionário proposto por Steglich. Outra peculiaridade observada nos artigos foi o número da amostra, no qual a grande maioria era composta pelo sexo feminino. 
O principal achado do presente estudo foi o efeito positivo da prática de atividades e/ou exercícios físicos sobre a autoimagem e autoestima em idosos de ambos os sexos. De acordo com o American College of Sports Medicine (2009), a prática regular de exercício físico é um dos meios efetivos para diminuir os efeitos negativos do envelhecimento tanto sobre os aspectos físicos como mentais.

Segundo Benedetti, Petroski e Gonçalves (2003), a convivência em grupo aliada a prática de exercício físico proporciona uma melhora consistente na autoestima e autoimagem corporal. $\mathrm{O}$ resultado do estudo vem ao encontro de pesquisas que vem discutindo o convívio e a participação social como um importante componente da autoestima (LUZ; AMATUZZI, 2008; MAZO; CARDOSO; AGUIAR, 2006), pois o momento de socialização ocorrido durante as práticas em grupo são importantes para os idosos, proporcionando prazer ao realizar as atividades (SEBIRE; STANDAGE; VANSTEENKISTE, 2009), bem como ao fortalecer o convívio social, fator imprescindível para o envelhecimento saudável, ao considerar o idoso um ser integrante da sociedade (GUEDES, M.; GUEDES, H.; ALMEIDA, 2011; SANTOS, 2001).

A percepção de melhoras na saúde, o aumento da sociabilidade e o prazer proporcionado pela prática do exercício físico são fatores que motivam os idosos a participar e permanecer nos grupos de convivência (SEBIRE; STANDAGE; VANSTEENKISTE, 2009; VITORELI; PESSINI; SILVA, 2005). No estudo de Guimarães e Caldas (2006), isso também foi observado. Os autores destacam a melhora do humor, redução do estresse, a melhora no funcionamento cognitivo, os efeitos positivos na imagem corporal e a melhora na qualidade do sono como fatores importantes para a evolução da qualidade de vida.

Roberts e Monroe (1994) afirmam que uma baixa autoestima está diretamente associada com fatores emocionais, estresse e conflitos interpessoais que, por sua vez, podem desencadear um quadro de depressão. Kadri et al. (2009) observaram quão motivadas estavam as pessoas com sintomas depressivos para a participação regular em um programa de caminhada nórdica e concluíram que há associação entre a presença de sintomas depressivos e baixa motivação para a prática de exercícios físicos. Outra hipótese que sustenta os resultados apresentados anteriormente é descrita por Sutoo e Akiyama (2003), na qual, do ponto de vista biológico, a atividade física e/ou exercício físico estão relacionados com a liberação de neurotransmissores ligados ao desempenho motor, motivação locomotora e modulação emocional (INGRAM, 2000).

A interferência das condições clínicas dos idosos em sua percepção de imagem corporal e autoestima é fato, que de maior ou menor proporção, pode 
comprometer o estilo de vida dos indivíduos acometidos por limitações sob tratamento, com complicações e restrições físicas ou mentais, sendo assim, idosos com menor número de doenças têm melhor autoestima que aqueles com maior acometimento por doenças (VITORELI; PESSINI; SILVA, 2005). A condição patológica requer dos idosos uma adaptação à nova imagem corporal, podendo esta ser lenta e, por vezes, difícil. Pois a qualidade de vida em situação de doença vai depender do controle da patologia com o tratamento adequado, ao considerar para além da dimensão fisiológica, os aspectos psicossociais vivenciados pelo idoso na percepção sobre sua própria realidade e autoaceitação (CAPISIANO, 1992).

Silva, Santos e Berardinelli (2010), apontam para a preocupação dos idosos com o descuidado com o corpo em suas dimensões física, intelectual/emocional e social espiritual, podem se apresentar como vulnerabilidades para as doenças crônicas. Assim, pensar em envelhecimento ativo e saudável inclui a preservação da capacidade para o autocuidado, ampliando a autonomia e consequentemente a autoestima do idoso (DUARTE, 1998). O autocuidado com o corpo exige do indivíduo conhecimentos e também a observação de si próprio, para a tomada de consciência daquilo que lhe faz mal nos seus hábitos de vida, de agir e de pensar (SILVA; SANTOS; BERARDINELLI, 2010).

\section{Considerações finais}

A análise dos 14 artigos que constituíram essa revisão evidenciou que existem variações consideráveis na forma como o idoso percebe e valoriza o próprio corpo, com destaque para a influência positiva da prática de atividades e/ou exercícios físicos na imagem corporal e autoestima dos idosos. A participação em grupos de prática de atividades físicas apresenta-se como um fator que pode contribuir para a melhoria da autoestima do idoso, visto que afeta positivamente sua capacidade física, a qual impacta de forma importante na preservação ou melhora da autonomia com desdobramentos positivos em todas as dimensões da vida. Além do mais, a dimensão social, bem como a socialização proporcionada pela da prática de atividades físicas em grupo apresenta-se como um importante da construção da imagem corporal e autoestima.

Outro aspecto importante evidenciado nos artigos analisados refere-se ao fato de que a população feminina apresenta níveis inferiores de satisfação da imagem corporal e autoestima em comparação com a população masculina. Cabe destacar que as mulheres constituíram a grande maioria dos sujeitos avaliados nos diferentes estudos. 
A autopercepção corporal e autoestima dos idosos ficam comprometidas com o adoecimento e sob as condições de tratamento a que são submetidos esses sujeitos. Nesse sentido, cabe ao profissional de saúde ou cuidador que trabalha com essa população, desenvolver estratégias de atenção e cuidado para resgatar a autoestima a melhorar a satisfação com a imagem corporal dos idosos nessa condição.

Por fim, cabe ressaltar que, apesar do aumento significativo de publicações relacionados à velhice e ao envelhecimento, verifica-se que poucos estudos abordam de forma clara a questão da imagem corporal e autoestima na população idosa, sendo esse o principal fator limitante de estudos de revisão nessa área. Dessa forma, ressaltamos a importância da produção científica sobre essa temática, fundamentalmente pelo seu potencial para subsidiar e instrumentalizar os profissionais para uma abordagem multidimensional e de qualidade na atenção à população idosa.

BODY IMAGE AND SELF-ESTEEM IN ELDERLY: AN INTEGRATIVE LITERATURE REVIEW

abstract

This paper presents an integrative review method aimed to analyze the scientific productions published on body image, and self-esteem in older people. The survey was carried out through direct research in full articles at the Biblioteca Virtual em Saúde using 24 articles, and resulting in a final sample of 14 articles, which met the criteria for inclusion in the study. The analysis allowed the subdivision of the selected papers in two categories: a) Physical exercise and its relationship with body image and self-esteem; b) Factors associated to the body image and self-esteem in older people. The results of this study showed the physical exercise as a positive influence on the perception of body image and self-esteem in older people. Among the factors associated to the body image and self-esteem it stands out higher dissatisfaction in female older people and the commitment of self-perception and self-esteem in older people in clinical conditions of disease and in health care. Thus, this review contributes to the scientific literature on body image and self-esteem in older people, subsidizing the actions of professionals for a multidimensional approach and quality care to the elderly. 
ALMEIDA, Graziela Aparecida Nogueira de; LOUREIRO, Sonia Regina; SANTOS, José Ernesto dos. A imagem corporal de mulheres morbidamente obesas avaliada através do desenho da figura humana. Psicologia: reflexão e crítica, Porto Alegre, v. 15, n. 2 , p. 283-292, maio/ago. 2002.

AMERICAN COLLEGE OF SPORTS MEDICINE (ACSM). Position stand on exercise and physical activity for older adults. Medicine and Science in Sports and Exercise, Indianápolis, v. 30, p. 1510-1530, jul. 2009.

ANTUNES, Giselli; MAZO, Giovana Zarpellon; BALBÉ, Giovane Pereira. Relação da autoestima entre a percepção de saúde e aspectos sociodemográficos de idosos praticantes de exercício físico. Revista da Educação Física da UEM, Maringá, v. 22, n. 4, p. 583-589, out./dez. 2011.

BENEDETTI, Tânia Bertoldo; PETROSKI, Édio Luiz; GONÇALVES, Lúcia Takase. Exercícios físicos, auto-imagem e auto-estima em idosos asilados. Revista Brasileira de Cineantropometria e Desempenho Humano, Florianópolis, v. 5, n. 2, p. 69-74, abr./jun. 2003.

BRANDÃO, Carmen Lúcia Coutinho et al. A imagem corporal do idoso com câncer atendido no ambulatório de cuidados paliativos do ICHC-FMUSP. Psicologia Hospitalar, São Paulo, v. 2, n. 2, dez. 2004

CAPISIANO, Helládio Francisco. Imagem corporal. In: MELLO FILHO, Júlio de et al. (Org.). Psicossomática hoje. Porto Alegre: Artes Médicas, 1992.

CHAIM, Janice; IZZO, Helena; SERA, Celisa Tiemi Nakagawa. Cuidar em saúde: satisfação com imagem corporal e autoestima de idosos. O Mundo da Saúde, São Paulo, v. 33, n. 2, p. 175-181, abr./jun. 2009.

DANTAS, Estélio. Pensando o corpo e o movimento. Rio de Janeiro: Shape, 1994.

DUARTE, Maria Jalma Rodrigues Santana. O envelhecer saudável: autocuidado para a qualidade de vida. Revista Enfermagem UERJ, Rio de Janeiro, v. 6, n. 1, p. 293-307, jun. 1998.

ERTHAL, Tereza Cristina. A auto-imagem: possibilidade e limitações da mudança. Arquivos Brasileiros de Psicologia, Rio de Janeiro, v. 38, n. 1, p. 39-46, jun./mar. 1986.

GANONG, Lawrence. Integrative reviews of nursing research. Research in Nursing \& Health, Malden, v. 10, n. 1, p. 1-11, fev. 1987.

GUEDES, Maria Helena Mota; GUEDES, Helisamara Mota; ALMEIDA, Martha Elisa Ferreira. Efeito da prática de trabalhos manuais sobre a autoimagem de idosos. Revista Brasileira de Geriatria e Gerontologia, Rio de Janeiro, v. 14, n. 4, p. 731-742, out./dez. 2011.

GUERRA, Ana Carolina Lima Cavaletti; CALDAS, Célia Pereira. Dificuldades e recompensas no processo de envelhecimento: a percepção do sujeito idoso. Ciência \& Saúde Coletiva, Rio de Janeiro, v. 15, n. 6, p. 2931-2940, set. 2010.

GUIMARÃES, Joanna Miguez Nery; CALDAS, Célia Pereira. A influência da atividade física nos quadros depressivos de pessoas idosas: uma revisão sistemática. Revista Brasileira de Epidemiologia, São Paulo, v. 9, n. 4, p. 481-492, dez. 2006. 
INGRAM, Donald. Age-related decline in physical activity: generalization to nonhumans. Medicine and Science in Sports and Exercise, Indianapolis, v. 32, n. 9, p. 1623-1629, set. 2000 .

KADRI, Sujia et al. Physical activity of depressed patients and their motivation to exercise: nordic walking in family practice. International Journal of Rehabilitation Research, Londres, v. 32, n. 2, p. 132-138, jun. 2009.

LUZ, Márcia Maria Carvalho; AMATUZZI, Mauro Martins. Vivências de felicidade de pessoas idosas. Estudos de Psicologia, Campinas, v. 25, n. 2, p. 303-7, jun. 2008.

MAGALHÃES, Carlos Henrique de Toledo et al. Auto-estima na forma inativa da oftalmopatia de Graves. Arquivo Brasileiro de Oftalmologia, São Paulo, v. 71, n. 2, p. 215-220, mar./abr. 2008.

MAZO, Giovana Zarpellon; CARDOSO, Fernando Luiz; AGUIAR, Daniela Lima. Programa de hidroginástica para idosos: motivação, auto-estima e auto-imagem. Revista Brasileira de Cineantropometria e Desempenho Humano, Florianópolis, v. 8, n. 2, p. 67-72, mar./abr. 2006

MENEZES, Tarciana Nobre de et al. Percepção da imagem corporal e fatores associados em idosos residentes em município do nordeste brasileiro: um estudo populacional. Ciência e Saúde Coletiva, Rio de Janeiro, v. 19, n. 8, p. 3451-3460, ago. 2014

MEURER, Simone Terezinha; BENEDETTI, Tânia Rosane Bertoldo; MAZO, Giovanna Zarpellon. Teoria da autodeterminação: compreensão dos fatores motivacionais e autoestima de idosos praticantes de exercícios físicos. Revista Brasileira de Atividade Física e Saúde, Pelotas v. 16, n. 1, p. 18-24, jan./mar. 2011

Aspectos da autoimagem e autoestima de idosos ativos. Motriz, Rio Claro, v. 15, n. 4, p. 788-796, out./dez. 2009

MEURER, Simone Terezinha et al. Associação entre sintomas depressivos, motivação e autoestima de idosos praticantes de exercícios físicos. Revista Brasileira de Ciências do Esporte, Porto Alegre, v. 34, n. 3, p. 683-695, jul./set. 2012.

NETTO, Matheus Papaléo. Gerontologia: a velhice e o envelhecimento em visão globalizada. São Paulo: Atheneu, 2002.

PINQUART, Martin; SÖRENSEN, Silvia. Gender differences in self-concept and psychological well-being in old age a meta-analysis. Journal of Gerontology: psychologica sciences, Washington, v. 56, n. 4, p. 195-213, jul. 2001.

PRACIDELLI, Fabiana et al. A imagem corporal dos idosos internados na enfermaria do Serviço de Geriatria do ICHCFMUSP - uma visão fisioterápica e psicológica. O Mundo da Saúde, São Paulo, v. 25, n. 4, p. 404-410, out./dez. 2001.

ROBERTS, John; MONROE, Scott. A multidimensional model of self-esteem in depression. Clinical Psychology Review, New York, v. 14, n. 3, p. 161-181, abr. 1994.

ROCHA, Fábio Lopes; CUNHA, Ulisses Gabriel Vasconcelos. Aspectos psicológicos e psiquiátricos das quedas do idoso. Arquivos Brasileiros de Medicina, Rio de Janeiro, v. 68, n. 1, p. 9-12, jan-fev. 1994.

ROCHA, Laura Maria B. C. Mariano da; TERRA, Newton. Body image in older adults: a review. Scientia Medica, Porto Alegre, v. 23, n. 4, out./dez. 2013.

ROSENBERG, Morris. Society and the adolescent self-image. Princeton: Princeton University Press, 1965

SAIKALI, Carolina Jabur et al. Imagem corporal nos transtornos alimentares. Revista de Psiquiatria Clínica, São Paulo, v. 31, n. 4, p. 164-166, jul./ago. 2004. 
Data de submissão: 03/12/2015

Data de aprovação: 14/04/2017
SANTOS, Aline Talita et al. Queixa subjetiva de comprometimento da memória em idosos saudáveis: influência de sintomas depressivos, percepção de estresse e autoestima. Revista da Escola de Enfermagem da USP, São Paulo, v. 46, n. esp., p. 24-29, out. 2012.

SANTOS, Silvana Sidney Costa. Enfermagem gerontogeriátrica: reflexão à ação cuidativa. São Paulo: Robe, 2001.

SEBIRE, Saimon; STANDAGE, Martyn; VANSTEENKISTE, Maarten. Examining intrinsic versus extrinsic exercise goals: Cognitive, affective, and behavioral outcomes. Journal of Sport and Exercise Psychology, Champaign, v. 31, n. 2, p. 189-210, 2009.

SILVA, Aila Cristina; SANTOS, Iraci; BERARDINELLI, Lina Márcia Miguéis. A imagem corporal do idoso no reflexo do autocuidado no envelhecimento saudável: estudo sociopoético. Online Brazilian Journal of Nursing, Rio de Janeiro, v. 9, n. 1, abr. 2010.

SILVA, Giulyanne Maria Lima da; CAMINHA, Iraquitan de Oliveira; GOMES, Isabelle Sena. O corpo e o tempo: a percepção dos idosos de um grupo de convivência. Pensar a Prática, Goiânia, v. 16, n. 4, out./dez. 2013.

SUTOO, Den'etsu; AKIYAMA, Kayo. Regulation of brain function by exercise. Neurobiology of Disease, New York, v. 13, n. 1, p. 1-14, jun. 2003.

VITORELI, Eliane; PESSINI, Salete; SILVA, Maria Júlia Paes. A auto-estima de idosos e as doenças crônico-degenerativas. Revista Brasileira de Ciências do Envelhecimento Humano, Passo Fundo, v. 2, n. 1, jan./jun. 2005. 